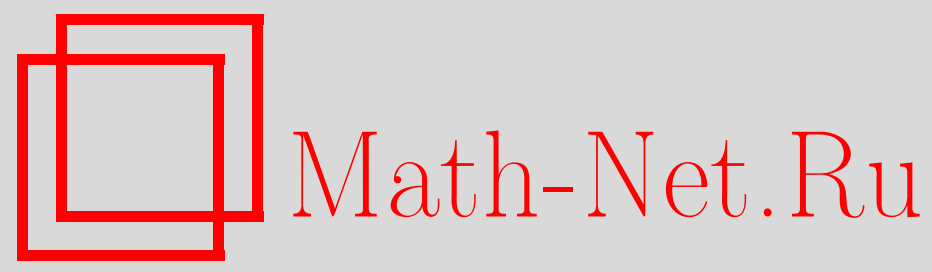

А. Ю. Чеботарев, Вариационные неравенства для оператора типа Навье-Стокса и односторонние задачи для уравнений вязкой теплопроводной жидкости, Матем. заметки, 2001, том 70, выпуск 2, 296-307

DOI: https://doi.org/10.4213/mzm742

Использование Общероссийского математического портала Math-Net.Ru подразумевает, что вы прочитали и согласны с пользовательским соглашением http://www. mathnet.ru/rus/agreement

Параметры загрузки:

IP : 54.80.97.219

26 апреля 2023 г., 16:34:07

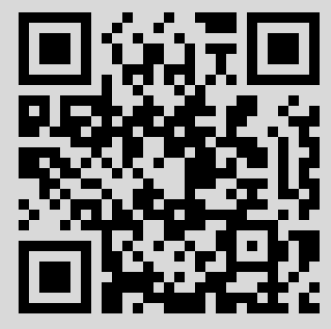




\title{
ВАРИАЦИОННЫЕ НЕРАВЕНСТВА ДЛЯ ОПЕРАТОРА ТИПА НАВЬЕ-СТОКСА И ОДНОСТОРОННИЕ ЗАДАЧИ ДЛЯ УРАВНЕНИЙ ВЯЗКОЙ ТЕПЛОПРОВОДНОЙ ЖИДКОСТИ
}

\author{
А. Ю. Чеботарев
}

Рассматривается класс стационарных вариационньг неравенств для операторов типа Навье-Стокса, в виде которых можно представить задачи с нелинейными краевьми условиями для уравнений динамики вязкой жидкости. Основной резултат - теорема о разрешимости - применяется для исследования односторонних краевых задач для системы уравнений тепловой конвекции вязкой жидкости.

Библиографоия: 19 названий.

\section{1. Введение}

Моделирование нелинейных условий типа неравенств в задачах гидродинамики приводит к вариационным неравенствам для соответствуюших гидродинамических операторов [1]-[9]. Классические краевые условия (например, условия Дирихле или Неймана) являются при этом частным случаем субдифференщиальных соотношений, связывающих гидродинамические параметры; с другой стороны, рассмотрение субдифференциальных краевых задач позволяет исследовать широкий класс физически интересных постановок. В работах [1]-[9] рассматривались вариационные неравенства для моделей гидродинамики, не учитьвающих тепловых эффектов. Учет влияния теплопроводности жидкости на ее физические характеристики приводит в рамках модели Буссинеска к появлению дополнительных нелинейных операторов по сравнению с классической моделью Навье-Стокса. В настоящей работе рассматривается класс стационарных неравенств для операторов типа Навье-Стокса, связанных с моделью динамики вязкой несжимаемой теплопроводной жидкости (модель тепловой конвекции). Естественно ожидать, что результаты для таких вариационных неравенств будут аналогичны соответствующим результатам для неравенств в модели однородной несжимаемой вязкой жидкости без учета тепловых эффектов, поскольку характер нелинейности соответствующих операторов не изменяется.

Результат о разрешимости вариационных неравенств для операторов типа НавьеСтокса применяется в данной работе к односторонним краевым задачам для системы

Работа выполнена при финансовой поддержке Российского фонда фундаментальных исследований, грант № 96-01-00256. 
Буссинеска. Отметим, что указанным способом можно также исследовать задачи тепловой конвекции вязкой жидкости с краевьми условиями, не содержащими неравенств, рассмотренными в [10]-[19], а также с различными нелинейньми краевыми условиями на скорость и температуру, аналогичными рассмотренным в [17].

\section{2. Вариационные неравенства для операторов типа Навье-Стокса}

Пусть $Y$ и $Z$ - вещественные гильбертовы пространства такие, что $Y \subset Z, Y$ плотно в $Z$ и вложение $Y$ в $Z$ компактно. Отождествляя $Z$ с его сопряженньм и обозначая через $Y^{\prime}$ сопряженное к $Y$, можно рассматривать $Z$ как подпространство в $Y^{\prime}, Y \subset Z \subset Y^{\prime}$. Обозначим через $\|\cdot\|,|\cdot|,\|\cdot\|_{*}$ нормы в пространствах $Y, Z, Y^{\prime}$ соответственно, а через $(f, y)$ - значение функционала $f \in Y^{\prime}$ на элементе $y \in Y$, совпадающее со скалярньп произведением в $Z$, если $f \in Z,((\cdot, \cdot))$ - скалярное произведение в пространстве $Y$.

ОПредЕЛЕниЕ. Оператор $N: Y \rightarrow Y^{\prime}$ будем называть оператором типа HaвьеСтокса, если

$$
N y=A y+B[y]+T y
$$

где

1) $A: Y \rightarrow Y^{\prime}$ - линейньй непрерывный оператор такой, что

$$
(A y, z)=(A z, y) \quad \exists \alpha>0: \quad(A y, y) \geqslant \alpha\|y\|^{2} \quad \forall y, z \in Y .
$$

2) $B[y]=B(y, y), B: Y \times Y \rightarrow Y^{\prime}$ - билинейньй непрерывньй оператор, удовлетворяющий условиям

$$
(B(y, z), z)=0 \quad \forall y, z \in Y
$$

отображение $B[y]: Y \rightarrow Y^{\prime}$ слабо непрерьвно.

3) $T: Y \rightarrow Y^{\prime}-$ компактньй оператор.

Пусть $\Phi: Y \rightarrow \overline{\mathbb{R}}=(-\infty ;+\infty]$ - вьпуклый, полунепрерьвньй снизу функционал, являющийся собственным в следующем смысле:

$$
\exists y_{0} \in Y \quad \Phi\left(y_{0}\right)<+\infty, \quad \forall y \in Y \quad \Phi(y)>-\infty .
$$

Через $\partial \Phi(y)$ обозначаем субдифференциал $\Phi$ в точке $y$, т.е. множество

$$
\partial \Phi(y)=\left\{g \in Y^{\prime}: \Phi(z)-\Phi(y) \geqslant(g, z-y) \quad \forall z \in Y\right\} .
$$

Главным объектом исследования в данном разделе будет следующее включение (уравнение с многозначньг оператором)

$$
-(N y-F) \in \partial \Phi(y) .
$$

Здесь $F \in Y^{\prime}$ - заданный элемент.

В силу определения $\partial \Phi(y)$ включение (4) равносильно вариационному неравенству

$$
(N y-F, y-z)+\Phi(y)-\Phi(z) \leqslant 0 \quad \forall z \in Y,
$$

при этом $\Phi(y)<+\infty$.

Как будет показано ниже в разделе 3 , в виде (4) или (5) можно записать ряд нелинейных краевых задач для уравнений динамики вязкой теплопроводной жидкости. Для доказательства разрешимости вариационного неравенства (5) воспользуемся результатами о существовании решений неравенств с псевдомонотонньпи операторами [1, с. 190]. 
Лемма 1 [1]. Oператор типа Навье-Стокса $N$ является псевдомонотонным.

ДокАЗАТЕЛЬСтво вытекает из слабой непрерывности отображения $y \rightarrow B[y]+T y$ и монотонности оператора $A[1$, с. 201].

Пусть $K \subset Y$ - замкнутое вьпуклое множество, являющееся эффективным множеством функционала $\Phi$; при этом

$$
\begin{gathered}
\Phi(y)=G(y)+I_{K}(y), \quad \text { где } I_{K}(y)-\text { индикаторная функция множества } K \\
I_{K}(y)= \begin{cases}0, & y \in K, \\
+\infty, & y \notin K,\end{cases} \\
G-\text { выпукльй и непрерьвньй на } K \text { функционал. }
\end{gathered}
$$

Лемма 2. Для произвольного $r>0$ существует решение $y \in K_{r}$ вариачионного неравенства

$$
(N y-F, y-z)+G(y)-G(z) \leqslant 0 \quad \forall z \in K_{r} .
$$

3дecb

$$
K_{r}=\{z \in K:\|z\|+|G(z)| \leqslant r\} .
$$

ДокАЗАТЕЛЬСтво. Следуя [1, с. 266], определим

$$
\begin{array}{cl}
\tilde{Y}=Y \times \mathbb{R}, \quad \tilde{K}=\{\tilde{z} \in \tilde{Y} ; \quad \tilde{z}=\{z, \alpha\}, \quad z \in K, \quad \alpha \geqslant G(z)\}, \\
\tilde{N} \tilde{y}=\{N y, 0\}, \quad \text { где } \tilde{y}=\{y, \xi\} \in \tilde{Y} .
\end{array}
$$

Оператор $\widetilde{N}$ является псевдомонотонным, а неравенство (8) равносильно неравенству

$$
(\tilde{N} \tilde{y}-\widetilde{F}, \tilde{y}-\tilde{z}) \leqslant 0 \quad \forall \tilde{z} \in \widetilde{K}_{r}
$$

где $\widetilde{K}_{r}=\{\tilde{z} \in \widetilde{K}: \tilde{z}=\{z, \alpha\},\|z\|+|\alpha| \leqslant r\}, \widetilde{F}=\{F,-1\}$. В силу ограниченности $K_{r}$ вариационное неравенство (9) имеет по крайней мере одно решение [1, с. 258].

ТЕорема 1. Пусть для достаточно больших $r>0$ произвольное решение неравенства (8) удовлетворяет априорной оценке

$$
\|y\| \leqslant C
$$

где $C$ не зависит от $y, r$. Тогда множество решений вариачионного неравенства (5) неnyсто. 
ДокАЗАТЕльСтво. Получим оценку решения $\tilde{y}$ неравенства (9), соответствующего решению $y \in K_{r}$ неравенства (8). Положим в $(9) \tilde{z}=\left\{y, G\left(y_{0}\right)\right\}, y_{0} \in K$. Тогда для $\tilde{y}=\{y, \xi\}$ имеем неравенства

$$
G(y) \leqslant \xi \leqslant G\left(y_{0}\right) .
$$

В силу условия (7) множество $\partial G\left(y_{0}\right)$ непусто и, следовательно,

$$
G(y) \geqslant G\left(y_{0}\right)+\left(\chi, y-y_{0}\right), \quad \chi \in \partial G\left(y_{0}\right) .
$$

Таким образом, из (10)-(12) получаем

$$
\begin{gathered}
G\left(y_{0}\right)-\|\chi\|_{*}\left(C+\left\|y_{0}\right\|\right) \leqslant \xi \leqslant G\left(y_{0}\right), \\
\|y\|+|\xi| \leqslant C_{1}=C+\left|G\left(y_{0}\right)\right|+\|\chi\|_{*}\left(C+\left\|y_{0}\right\|\right) .
\end{gathered}
$$

Отметим, что постоянная $C_{1}$ не зависит от $r$. Выбирая $r>C_{1}$, получаем, что $\tilde{y}$ фактически является решением неравенства

$$
(\tilde{N} \tilde{y}-\widetilde{F}, \tilde{y}-\widetilde{w}) \leqslant 0 \quad \forall \widetilde{w} \in \widetilde{K}
$$

Действительно, если $\widetilde{w} \in \widetilde{K}$ произвольный элемент, то при малых $\varepsilon>0$

$$
\tilde{z}=(1-\varepsilon) \tilde{y}+\varepsilon \widetilde{w} \in K_{r} \quad \text { при } r>C_{1} \text {. }
$$

Тогда из (9) следует (13). Поскольку (13) эквивалентно вариационному неравенству (5), то теорема доказана.

Следуя [5], можно привести достаточные условия на операторы $B, T$ и множество ограничений $K$, гарантирующие получение априорной оценки (10). В частности, достаточно обосновать “подчиненность" нелинейного оператора $B[u]+T(u)$ оператору $A$ на множестве $K$; а именно, для всех $\gamma>0$ существует $w_{\gamma} \in K$ такое, что

$$
\left(B[y], w_{\gamma}\right)-\left(T y, y-w_{\gamma}\right) \leqslant \gamma\|y\|^{2}+C_{\gamma} \quad \forall y \in K
$$

где $C_{\gamma}$ не зависит от $y \in K$.

\section{3. Разрешимость односторонних краевых задач для уравнений тепловой конвекции}

3.1. Постановка краевых задач. Пусть $\Omega$-ограниченная односвязная область в пространстве $\mathbb{R}^{3}$ со связной границей $\Gamma \in C^{2}$. Рассмотрим движение вязкой теплопроводной жидкости в области $\Omega$, которая описывается следующей системой уравнений:

$$
\begin{gathered}
-\nu \Delta u+\operatorname{rot} u \times u+\beta g \theta=-\nabla h+f, \quad \operatorname{div} u=0, \\
-\varkappa \Delta \theta+u \nabla \theta=q .
\end{gathered}
$$

Здесь $u=\left\{u_{i}\right\}_{1}^{3}, h$ и $\theta-$ скорость, полный напор и температура жидкости, $\nu=$ const $>0-$ коэффициент кинематической вязкости, $\varkappa=$ const $>0$ - коэффициент температуропроводности, $g=-\left(0,0, g_{0}\right)$ - вектор ускорения свободного падения (при этом ось $x_{3}$ 
декартовой системы координат считается направленной вдоль вектора $(-g)), f$ - массовая плотность внешних сил, $q$ - объемная плотность источников тепла.

Предположим, что на гранище Г заданы нулевые касательные компоненты вектора скорости

$$
u_{\tau}=u-u_{n} n=0,
$$

где $n$ - единичный вектор внешней нормали к границе $\Gamma, u_{n}=(u \cdot n)$.

Субдифференциальная краевая задача для системы $(14),(15)$ заключается в отыскании решения этой системы, удовлетворяющего условию (16) и субдифференциальньп граничным условиям

$$
\begin{gathered}
h \in \partial \varphi\left(u_{n}, x\right), \\
-\frac{\partial \theta}{\partial n} \in \partial \Psi(\theta, x), \quad x \in \Gamma .
\end{gathered}
$$

Здесь через $\varphi: \mathbb{R} \times \Gamma \rightarrow \overline{\mathbb{R}}=(-\infty,+\infty], \Psi: \mathbb{R} \times \Gamma \rightarrow \overline{\mathbb{R}}$ обозначены заданные выпуклые и полунепрерьвные снизу по первому аргументу функции, $\varphi \not \equiv+\infty, \Psi \not \equiv+\infty$. Под $\partial \varphi(a, x)$ (соответственно $\partial \Psi(a, x))$ понимается субдифференциал $\varphi$ (соответственно $\Psi$ ) по первому аргументу, т.е. множество

$$
\partial \varphi(a, x)=\{z \in \mathbb{R}: \varphi(b, x)-\varphi(a, x) \geqslant z(b-a) \quad \forall b \in \mathbb{R}\} .
$$

Наличие второго аргумента $x$ в функциях $\varphi, \Psi$ связанно с тем, что граничные условия (17), (18) могут иметь различньй характер на разных участках границы $\Gamma$.

Приведем примеры односторонних краевых условий, которые можно записать в виде (17), (18).

ПРимеР 1. Пусть заданы две функции $\theta_{1}(x), \theta_{2}(x), x \in \Gamma, 0<\theta_{1}(x) \leqslant \theta_{2}(x)$, и требуется, чтобы температура $\theta(x)$ на гранище Г принимала значения из отрезка $\left[\theta_{1}(x), \theta_{2}(x)\right]$. Определим функцию $\Psi$ следуюшим образом:

$$
\Psi(\lambda, x)= \begin{cases}0, & \lambda \in\left[\theta_{1}(x), \theta_{2}(x)\right] \\ +\infty & \text { иначе. }\end{cases}
$$

Тогда

$$
\partial \Psi(\lambda, x)= \begin{cases}{[0,+\infty),} & \lambda=\theta_{2}(x) \\ 0, & \lambda \in\left(\theta_{1}(x), \theta_{2}(x)\right) ; \\ (-\infty, 0], & \lambda=\theta_{1}(x) .\end{cases}
$$

Условие (18) в данном случае принимает вид односторонних граничных условий на температуру:

$$
\begin{gathered}
\theta_{1}(x) \leqslant \theta(x) \leqslant \theta_{2}(x), \quad \frac{\partial \theta}{\partial n}=0, \quad \text { если } \theta_{1}(x)<\theta(x)<\theta_{2}(x), \\
\frac{\partial \theta}{\partial n} \geqslant 0, \text { если } \theta(x)=\theta_{1}(x), \quad \frac{\partial \theta}{\partial n} \leqslant 0, \quad \text { если } \theta=\theta_{2}(x) .
\end{gathered}
$$

Далее, выбрав в качестве $\varphi$ функцию

$$
\varphi(\lambda, x)= \begin{cases}0, & \text { если } \lambda=0, \\ +\infty & \text { иначе, }\end{cases}
$$

мы дополним краевые условия (20) условием Дирихле для скорости течения

$$
u=0, \quad x \in \Gamma \text {. }
$$


ПримеР 2. Пусть граница Г области течения $\Omega$ состоит из участков $\Gamma_{0}, \Gamma_{1}, \Gamma_{2}$ таких, что

$$
\begin{aligned}
u=0, & x \in \Gamma_{0}-\text { непроницаемая часть границы, } \\
u_{n} \leqslant 0, & x \in \Gamma_{1}-\text { участок "втекания", } \\
u_{n} \geqslant 0, & x \in \Gamma_{2}-\text { участок “вытекания", } \\
& \Gamma=\Gamma_{0} \cup \Gamma_{1} \cup \Gamma_{2} .
\end{aligned}
$$

Зададим функцию $\varphi$ соотношением

$$
\varphi(\lambda, x)= \begin{cases}l(x) \lambda, & \text { если } x \in \Gamma_{1}, \lambda \leqslant 0 \text { или } x \in \Gamma_{2}, \lambda \geqslant 0, \\ 0, & \text { если } x \in \Gamma_{0}, \\ +\infty & \text { иначе },\end{cases}
$$

где $l(x), x \in \Gamma_{1} \cup \Gamma_{2},-$ заданная функция. В этом случае помимо односторонних граничных условий для температуры (20) из (17) вытекают односторонние условия для скорости и полного напора

$$
\begin{gathered}
u_{n}(h-l)=0, \quad h \geqslant l, \quad u_{n} \geqslant 0, \quad x \in \Gamma_{1}, \\
h \leqslant l, \quad u_{n} \leqslant 0, \quad x \in \Gamma_{2}, \quad u_{n}=0, \quad x \in \Gamma_{0} .
\end{gathered}
$$

ЗАМЕчАниЕ 1. Полагая

$$
\varphi_{(\lambda, x)}=\left\{\begin{array}{ll}
0, & \lambda=u_{0}(x), \\
+\infty & \text { иначе },
\end{array} \quad \Psi(\lambda, x)= \begin{cases}0, & \lambda=\theta_{0}(x), \\
+\infty & \text { иначе }\end{cases}\right.
$$

можно записать в виде $(17),(18)$ классические граничные условия

$$
u_{n}=u_{0}(x), \quad \theta=\theta_{0}(x), \quad x \in \Gamma .
$$

ЗАмЕЧАниЕ 2. Можно рассмотреть обобщение "разделенных" условий (17), (18), если обозначить

$$
y=\left(\begin{array}{c}
u \\
\theta
\end{array}\right), \quad z=\left(\begin{array}{c}
h \\
-\partial \theta / \partial n
\end{array}\right), \quad \gamma y=\left(\begin{array}{c}
u_{n} \\
\theta \mid \Gamma
\end{array}\right)
$$

и определить вьпуклую функцию $\xi(\mu, x): \mathbb{R}^{2} \times \Gamma \rightarrow(-\infty,+\infty]$, полунепрерьвную снизу по $\mu$, причем $\xi \not \equiv+\infty$. Тогда субдифференциальное условие

$$
z \in \partial \xi(\gamma y, x), \quad x \in \Gamma
$$

позволяет описывать различные, более сложные краевые условия, связывающие скорость и температуру жидкости на границе $\Gamma$. 
3.2. Обобщенное решение задачи (14)-(18). Обозначим через $\mathscr{W}$ множество бесконечно-дифференцируемых в $\Omega$, соленоидальных вектор-функций $w$ таких, что

$$
w \in C^{\infty}(\bar{\Omega}), \quad w_{\tau}=0, \quad x \in \Gamma .
$$

Через $V, H$ обозначим замыкание $\mathscr{W}$ в нормах $W_{2}^{1}(\Omega)$ и $L^{2}(\Omega)$ соответственно. Здесь и далее через $W_{p}^{l}$ обозначается обычное пространство Соболева. В качестве скалярных произведений в пространствах $V, H$ выберем билинейные формы

$$
(u, v)_{V}=\int_{\Omega} \operatorname{rot} u \cdot \operatorname{rot} v d x ; \quad(u, v)_{H}=(u, v)=\int_{\Omega} u \cdot v d x .
$$

Отметим, что норма в $V$, порождаемая указанным скалярньм произведением, эквивалентна норме в $W_{2}^{1}(\Omega)$, что вытекает из равенства [3, с. 138$]$

$$
(\operatorname{rot} u, \operatorname{rot} v)=(\nabla u, \nabla v)+\int_{\Gamma} k\left(u_{n} v_{n}\right) d s,
$$

справедливого для соленоидальных векторов $u, v \in W_{2}^{1}(\Omega)$ с нулевыми касательными составляющими на границе $\Gamma$. Здесь $k=k(x)$ - удвоенная средняя кривизна $Г$ в точке $x$.

Пусть

$$
Y=V \times W_{2}^{1}(\Omega), \quad Y_{0}=H \times L^{2}(\Omega),
$$

причем вторые сомножители в декартовом произведении пространств являются пространствами скалярных функций; при этом

$$
(y, z)_{Y}=((u, v))+(\theta, \zeta)_{W_{2}^{1}(\Omega)} \quad \forall y=\{u, \theta\}, \quad z=\{v, \zeta\}
$$

Отождествляя $Y_{0}$ с его сопряженным $Y_{0}^{\prime}$, имеем

$$
Y \subset Y_{0}=Y_{0}^{\prime} \subset Y^{\prime} .
$$

Через $(F, y)$ будем обозначать значение функционала $F \in Y^{\prime}$ на элементе $y \in Y$, которое совпадает со скалярньм произведением в $Y_{0}$, если $F, y \in Y_{0}$,

$$
(F, y)=(f, u)+\int_{\Omega} \zeta(x) \theta(x) d x, \quad F=\{f, \zeta\}, \quad y=\{u, \theta\} .
$$

Определим операторы $A: Y \rightarrow Y^{\prime}, B: Y \times Y \rightarrow Y^{\prime}$ и функционал $\Phi: Y \rightarrow \overline{\mathbb{R}}=(-\infty ;+\infty]$ для всех $y=\{u, \theta\}, z=\{v, \zeta\}, r=\{w, \xi\}$ следующими соотношениями:

$$
\begin{gathered}
(A y, z)=\nu(\operatorname{rot} u, \operatorname{rot} v)+\varkappa(\nabla \theta, \nabla \zeta), \\
(B(y, z), r)=\int_{\Omega}(\operatorname{rot} u \times v) w d x, \\
(T y, z)=\beta \int_{\Omega} g \theta v d x+\int_{\Omega} u \nabla \theta \zeta d x, \\
\Phi(y)=\left\{\begin{array}{lc}
\int_{\Gamma}\left(\varphi\left(u_{n}, x\right)+\psi(\theta, x)\right) d s, & \text { если } \varphi\left(u_{n}(x), x\right)+\psi(\theta(x), x) \in L^{1}(\Gamma), \\
+\infty & \text { иначе. }
\end{array}\right.
\end{gathered}
$$

Пусть

$$
F=\{f, q\} \in Y^{\prime}, \quad(F, z)=\int_{\Omega}(f v+q \zeta) d x,
$$

если $f \in L^{2}(\Omega), q \in L^{2}(\Omega)$. 
ОПредЕЛЕниЕ. Элемент $y=\{u, \theta\} \in Y$ назьвается обобщенным решением задаи (14)-(18), если для всех $z \in Y$ вьполнено неравенство

$$
(A y+B[y]+T y-F, y-z)+\Phi(y)-\Phi(z) \leqslant 0 .
$$

Вариационное неравенство (31) получается путем умножения уравнения импульсов в $(14)$ на $(u-v)$, уравнения для температуры на $(\theta-\varphi)$, интегрирования по частям в $\Omega$ и сложения неравенств, полученных после применения условий (17), (18). Покажем, что в определенном смысле справедливо и обратное. Если $y=\{u, \theta\}$, где функции $u, \theta$ являются достаточно гладкими, то найдется скалярная функция $h$ (полньй напор течения) такая, что тройка $u, \theta, h$ удовлетворяет условиям (14)-(18). Действительно, поскольку $u \in V$, то вьполняется условие (16) и уравнение неразрьвности $\operatorname{div} u=0$, что вытекает из определения пространства $V$. Полагая в неравенстве (31) для произвольного $w \in V \cap C_{0}^{\infty}(\Omega)$

$$
y=\{u, \theta\}, \quad z=\{u \pm w, \theta\}
$$

получаем

$$
\nu(\operatorname{rot} u, \operatorname{rot} w)+\int_{\Omega}(\operatorname{rot} u \times u) w d x+\beta(\theta g, w)-(f, w)=0 .
$$

Последнее равенство влечет выполнение уравнения импульсов в (14) для некоторой скалярной функции $h[18$, с. 27$]$.

Умножив указанное уравнение на $(u-v)$, проинтегрировав по $\Omega$ и сравнив затем с неравенством (31), где в качестве $z \in Y$ выбрана пара $\{v, \theta\}$, получаем

$$
\int_{\Gamma}\left(\varphi\left(v_{n}, x\right)-\varphi\left(u_{n}, x\right)\right) d s \geqslant \int_{\gamma} h\left(v_{n}-u_{n}\right) d s .
$$

Следовательно [19, с. 134], справедливо условие (17). Аналогичным образом, полагая в $(31) z=\left\{u, \theta \pm \zeta_{0}\right\}$, где $\zeta_{0} \in C_{0}^{\infty}(\Omega)$, получаем уравнение $(15)$ и включение $(18)$.

\section{3. Разрешимость односторонних краевых задач.}

Лемма 3. Onepamop $N: Y \rightarrow Y^{\prime}$, где $N y=A y+B[y]+T y$, a onepamop $T$ определены в (27)-(29), является оператором типа Навье-Стокса.

ДокАЗАтЕльСтво. Проверка условий (1), (2) очевидна. Условие (3) проверяется аналогично [5]. Осталось доказать компактность оператора (29). Пусть $y_{m}=\left\{u_{m}, \theta_{m}\right\}$ - ограниченная в $Y$ последовательность. Тогда, в случае необходимости переходя к подпоследовательности, заключаем

$$
\begin{gathered}
u_{m} \rightarrow u \text { слабо в } V, \text { сильно в } H, L^{4}(\Omega), \\
\theta_{m} \rightarrow \theta \text { слабо в } W_{2}^{1}(\Omega), \text { сильно в } L^{2}(\Omega), L^{4}(\Omega), L^{2}(\Gamma) .
\end{gathered}
$$

Пусть $z=\{v, \xi\},\|z\|_{Y}=1, y=\{u, \theta\}$. Рассмотрим

$$
\begin{aligned}
\left(T y_{m}-T y, z\right)= & \beta \int_{\Omega} g\left(\theta_{m}-\theta\right) v d x+\int_{\Omega}\left(u_{m} \nabla \theta_{m}-u \nabla \theta\right) \xi d x \\
\leqslant & \beta|g|\left\|\theta_{m}-\theta\right\|_{L^{2}(\Omega)}\|V\|_{H}+\left\|u_{m}-u\right\|_{L^{4}(\Omega)}\left\|\nabla \theta_{m}\right\|_{L^{2}(\Omega)}\|\xi\|_{L^{4}(\Omega)} \\
& \quad+\|u\|_{L^{4}(\Omega)}\|\nabla \xi\|_{L^{2}(\Omega)}\left\|\theta_{m}-\theta\right\|_{L^{4}(\Omega)}+\int_{\Gamma}(u \cdot n)\left(\theta_{m}-\theta\right) \xi d s .
\end{aligned}
$$


Тогда

$$
\begin{aligned}
\left\|T y_{m}-T y\right\|_{y^{\prime}} & =\sup _{\|z\|=1}\left(T y_{m}-T y, z\right) \\
& \leqslant C_{m}\left(\left\|\theta_{m}-\theta\right\|_{L^{2}(\Omega)}+\left\|u_{m}-u\right\|_{L^{4}(\Omega)}+\left\|\theta_{m}-\theta\right\|_{L^{4}}+\left\|\theta_{m}-\theta\right\|_{L^{2}(\Gamma)}\right) .
\end{aligned}
$$

Здесь

$$
C_{m}=\max \left(\beta|g|\|v\|,\left\|\nabla \theta_{m}\right\|\|\xi\|_{L^{4}(\Omega)},\|u\|_{L^{4}(\Omega)}\|\nabla \xi\|_{L^{2}(\Omega)},\left\|u_{n}\right\|_{L^{4}(\Gamma)}\|\xi\|_{L^{4}(\Gamma)}\right)
$$

- последовательность, ограниченная в силу (33) и условий $\|v\| \leqslant 1,\|\xi\|_{W_{2}^{1}(\Omega)} \leqslant 1$. Из (34) на основании (32), (33) вытекает, что $T y_{m} \rightarrow T y$ сильно в $Y^{\prime}$. Поскольку непрерьвность $T$ очевидна, то лемма доказана.

Рассмотрим примеры приложения теоремы 1 к доказательству разрешимости односторонних краевых задач для уравнений тепловой конвекции.

ЗАДАчА 1. Найти решение системы $(14),(15)$, удовлетворяющее граничньп условиям (16), (20), (22).

Как показано в п. 3.1, в качестве функций $\varphi, \psi$ в граничных условиях (17), (18) выбираются функции $(21),(19)$ соответственно и при этом функция $\Phi$ является индикаторной функцией множества $K \subset Y$,

$$
K=\left\{y=\{u, \theta\}: u_{n}(x)=0, \theta_{1}(x) \leqslant \theta(x) \leqslant \theta_{2}(x), x \in \Gamma\right\}
$$

Таким образом, элемент $y \in K$ назьвается обобщенным решением задачи 1 , если

$$
(A y+B[y]+T y-F, y-z) \leqslant 0 \quad \forall z \in K
$$

ТЕОрема 2. Пусть выполняются следуюшие условия: $\theta_{1}, \theta_{2} \in L^{2}(\Gamma), 0<\theta_{1}(x) \leqslant$ $\theta_{0}(x) \leqslant \theta_{2}(x)$ почти всюду на $\Gamma, \theta_{0} \in W_{2}^{1}(\Omega), f \in L^{2}(\Omega), q \in L^{2}(\Omega)$. Тогда задача 1 разрешима в обобщенном смысле.

ДокАЗАТЕЛЬСтво. В силу леммы 2 для произвольного $r>0$ сушествует решение $y=\{u, \theta\}$ вариационного неравенства

$$
\begin{gathered}
(A y+B[y]+T y-F, y-z) \leqslant 0 \quad \forall z \in K_{r} \\
K_{r}=\left\{z \in K:\|z\|_{Y} \leqslant r\right\} .
\end{gathered}
$$

Получим априорную оценку решения неравенства (36). Рассмотрим семейство срезающих функций $[1$, с. 116$] \mu_{\varepsilon} \in C^{2}(\bar{\Omega})$ :

$$
\begin{array}{ll}
\mu_{\varepsilon}(x)=1 & \text { в окрестности } \Gamma=\partial \Omega, \text { зависящей от } \varepsilon, \\
\mu_{\varepsilon}(x)=0, & \text { если } \rho(x)=\operatorname{dist}(x, \Gamma) \geqslant \delta(\varepsilon), \delta(\varepsilon)=\exp (-1 / \varepsilon), \\
\left|\nabla \mu_{\varepsilon}(x)\right| \leqslant \varepsilon / \rho(x), & \text { если } \rho(x) \leqslant \delta(\varepsilon) .
\end{array}
$$


В (36) полагаем $z=\left\{u, \mu_{\varepsilon} \theta_{0}\right\}$, предполагая, что значение $r>0$ является достаточно большим, т.е.

$$
\left\|\mu_{\varepsilon} \theta_{0}\right\|_{W_{2}^{1}(\Omega)}^{2} \leqslant r^{2}-\|u\|^{2} .
$$

Выбор $r$, удовлетворяющего (37), будет указан ниже. Тогда

$$
\varkappa\left(\nabla \theta, \nabla\left(\theta-\mu_{\varepsilon} \theta_{0}\right)\right)+\left(u \nabla \theta, \theta-\mu_{\varepsilon} \theta_{0}\right) \leqslant\left(q, \theta-\mu_{\varepsilon} \theta_{0}\right) .
$$

Преобразуем второе слагаемое в левой части (38).

$$
\begin{aligned}
& \left(u \nabla \theta, \theta-\mu_{\varepsilon} \theta_{0}\right)=\left(u \nabla\left(\mu_{\varepsilon} \theta_{0}\right), \theta-\mu_{\varepsilon} \theta_{0}\right) \\
& \quad=\left(u \nabla \mu_{\varepsilon} \theta_{0}, \theta-\mu_{\varepsilon} \theta_{0}\right)+\left(u \nabla \theta_{0} \mu_{\varepsilon}, \theta-\mu_{\varepsilon} \theta_{0}\right)=I_{1}+I_{2} .
\end{aligned}
$$

На основании оценок

$$
\begin{aligned}
& \left|I_{1}\right| \leqslant\left\|\frac{u}{\rho}\right\|_{L^{2}(\Omega)}\left\|\theta_{0}\right\|_{L^{4}\left(\Omega_{\varepsilon}\right)}\left\|\theta-\mu_{\varepsilon} \theta_{0}\right\|_{L^{4}(\Omega)}, \\
& \left|I_{2}\right| \leqslant\|u\|_{L^{4}(\Omega)}\left\|\nabla \theta_{0}\right\|_{L^{2}\left(\Omega_{\varepsilon}\right)}\left\|\theta-\mu_{\varepsilon} \theta_{0}\right\|_{L^{4}(\Omega)},
\end{aligned}
$$

где $\Omega_{\varepsilon}=\{x \in \Omega: \rho(x) \leqslant \delta(\varepsilon)\}$, а также неравенств [1, с. 116]

$$
\left\|\frac{u}{\rho}\right\|_{L^{2}(\Omega)} \leqslant C\|u\|_{V}, \quad\|u\|_{L^{4}(\Omega)} \leqslant C\|u\|_{V},
$$

где $C>0$ не зависят от $u$, заключаем

$$
\left|\left(u \nabla \theta, \theta-\mu_{\varepsilon} \theta_{0}\right)\right| \leqslant \alpha\|u\|_{V}\left\|\theta-\mu_{\varepsilon} \theta_{0}\right\|_{L^{4}(\Omega)} .
$$

Здесь $\alpha=C\left(\left\|\theta_{0}\right\|_{L^{4}\left(\Omega_{\varepsilon}\right)}+\left\|\nabla \theta_{0}\right\|_{L^{2}\left(\Omega_{\varepsilon}\right)}\right) \rightarrow 0$ при $\varepsilon \rightarrow 0$. Воспользуемся далее неравенством

$$
\|\theta\|_{W_{2}^{1}(\Omega)}^{2} \leqslant C\left(\|\nabla \theta\|_{L^{2}(\Omega)}^{2}+\|\theta\|_{L^{2}(\Gamma)}^{2}\right) .
$$

Из оценок (38)-(40), с учетом неравенств вложения $W_{2}^{1}(\Omega) \rightarrow L^{p}(\Omega), p=2,4$, выводим для произвольного $\alpha>0$ оценку

$$
\|\theta\|_{W_{2}^{1}(\Omega)}^{2} \leqslant \alpha\|u\|^{2}+C_{\alpha}
$$

Здесь постоянная $C_{\alpha}>0$ зависит только от $\alpha$ и исходных данных задачи: $\Omega, q, \theta_{0}, \varkappa$. Выбрав в неравенстве $(36) z=\{0, \theta\}$, получим неравенство

$$
\nu\|u\|^{2}+\beta(\theta g, u) \leqslant(f, u) .
$$

Из оценок $(41),(42)$ в силу произвольности $\alpha>0$ легко вытекают следующие оценки решения неравенства (36):

$$
\|u\| \leqslant C_{1}, \quad\|\theta\|_{W_{2}^{1}(\Omega)} \leqslant C_{2}
$$

с постоянными $C_{1}, C_{2}$, зависящими только от исходных данных $\Omega, q, \theta_{0}, \varkappa, \nu, f, \beta, g$; при этом значение параметра $r>0$ достаточно взять большим, чем $\left(\left\|\mu_{\varepsilon} \theta_{0}\right\|_{W_{2}^{1}(\Omega)}^{2}+C_{1}^{2}\right)^{1 / 2}$. Применяя теорему 1 , на основании априорных оценок (43) приходим к утверждению теоремы 2.

Рассмотрим теперь одностороннюю задачу для уравнений тепловой конвекции, в которой ограничения в виде неравенств накладьваются на значение нормальной компоненты скорости и полного напора. 
ЗАДАчА 2. Найти решение системы $(14),(15)$, удовлетворяющее граничньм условиям (16), (20), (24).

В данном случае, выбирая $\varphi, \psi$ в соответствии с $(23),(19)$, мы получаем обобщенную формулировку задачи 2 в виде неравенства (31), где функционал $\Phi$ имеет вид

$$
\Phi(y)=\int_{\Gamma_{1} \cup \Gamma_{2}} l(x) u_{n}(x) d s+I_{K}(y)
$$

Здесь $I_{K}-$ индикаторная функция множества

$$
\begin{aligned}
K= & \left\{y \in Y: y=\{u, \theta\}, \quad u_{n} \leqslant 0, \quad x \in \Gamma_{1} ; \quad u_{n} \geqslant 0, \quad x \in \Gamma_{2}\right. \\
& \left.u_{n}=0, \quad x \in \Gamma_{0} ; \quad \theta_{1}(x) \leqslant \theta(x) \leqslant \theta_{2}(x), \quad x \in \Gamma\right\} .
\end{aligned}
$$

Априорные оценки, аналогичные оценкам, полученным при доказательстве теоремы 2 , приводят к следующему результату.

ТЕОрема 3. Пусть выполняются условия теоремы 2 и $l(x) \in L^{2}\left(\Gamma_{1} \cup \Gamma_{2}\right)$. Тогда существует по крайней мере одно обобщенное решение задачи 2.

ЗАмЕчАниЕ. Как было указано в п. 3.1, в форме (17), (18) можно записать условия Дирихле (25) для скорости и температуры, при этом обобщенной формулировкой соответствующей краевой задачи будет неравенство (31), где функционал $\Phi$ является индикаторной функцией множества

$$
K=\left\{y \in Y, \quad y=\{u, \theta\}: u_{n}=u_{0}(x), \quad \theta=\theta_{0}(x), \quad x \in \Gamma\right\}
$$

Для получения априорной оценки обобщенного решения задачи Дирихле достаточно повторить вьвод неравенства (41) для температуры $\theta$ и применить аналогичньй прием для вьвода оценки скорости, подставляя в неравенство (31) $z=\left\{\mu_{\varepsilon} w, \theta\right\}$, где $w \in V$ соленоидальное продолжение граничной функции $u_{0}$ в $\Omega, \mu_{\varepsilon}-$ срезка, определенная при доказательстве теоремы 2 . В результате получаем следующее утверждение.

ТеоремА 4. Пусть выполняются условия: $f \in L^{2}(\Omega), q \in L^{2}(\Omega), \theta_{0} \in H^{1 / 2}(\Gamma)$, $u_{0} \in H^{1 / 2}(\Gamma), \int_{\Gamma} u_{0} d s=0$. Тогда существует по крайней мере одно обобщенное решение краевой задачи (14)-(16), (25).

Здесь через $H^{1 / 2}(\Gamma)$ обозначается пространство следов на границе Г функций из $W_{2}^{1}(\Omega)$. 


\section{СПИСОК ЦИТИРОВАННОЙ ЛИТЕРАТУРЫ}

[1] Лионс ЖК.-Л. Некоторые методы решения нелинейных краевых задач. М.: Мир, 1972.

[2] Кажихов А. В. Разрешимость некоторых односторонних краевых задач для уравнений Навье-Стокса // Динамика сплошной среды. № 16. Новосибирск, 1974. С. 5-34.

[3] Антонцев С.Н., Кажихов А. В., Монахов В.Н. Краевые задачи механики неоднородных жидкостей. Новосибирск: Наука, 1983.

[4] Чеботарев А.Ю. Об односторонних и экстремальных задачах, связанных с системой Стокса // Динамика сплошной среды. № 102. Новосибирск, 1991. С. 133-147.

[5] Чеботарев А. Ю. Субдифференциальные краевые задачи для стационарных уравнений Навье-Стокса // Дифференц. уравнения. 1992. Т. 28. № 8. С. 1443-1450.

[6] Bre'zis H. Inéquations variationnelles relatives a l'operateur de Navier-Stokes // J. Math. Anal. Appl. 1972. V. 39. № 1. P. 159-165.

[7] Müller M., Naumann J. On evolution inequalities of a modified Navier-Stokes type. I, II, III // Aplikace Matematiky. 1978. V. 24. № 2. P. 174-184; №6. P. 397-407; V. 24. № 2. P. 81-91.

[8] Чеботарев А. Ю. Разрешимость стационарной односторонней задачи протекания для идеальной жидкости // Динамика сплошной среды. № 79. Новосибирск, 1987. С. 129-135.

[9] Чеботарев А. Ю. Стационарные вариационные неравенства в модели неоднородной несжимаемой жидкости // Сиб. матем. ж. 1997. Т. 38. № 5. С. 1184-1193.

[10] Уховский М. Ф., Юдович В. И. Об уравнениях стационарной конвекции // ПММ. 1963. Т. 27. № 2. С. $295-300$.

[11] Юдович В. И. О возникновении конвекции // ПММ. 1966. Т. 30. №6. С. 1000-1005.

[12] Юдович В. И. Свободная конвекция и ветвление // ПММ. 1967. Т. 31. № 1. С. 101-111.

[13] Зарубин А. Г. Задача стационарной тепловой конвекции // ЖВМиМФ. 1968. Т. 8. №6. C. $1378-1383$.

[14] Зарубин А. Г., Тиунчик М.Ф. Некоторые задачи механики с разрывными граничными условиями и негладкой границей // Дифференц. уравнения. 1978. Т. 14. № 9. С. 1632-1637.

[15] Алексеев Г.В. Теоретический анализ стационарных задач граничного управления для уравнений тепловой конвекции. Препринт ИПМ ДВО РАН. Владивосток: Дальнаука, 1996.

[16] Алексеев Г.В., Терешко Д.А. Обратные экстремальные задачи для стационарных уравнений тепловой конвекции. Препринт ИПМ ДВО РАН. Владивосток: Дальнаука, 1997.

[17] Дюво Г., Лионс ЖК.-Л. Неравенства в механике и физике. М.: Наука, 1980.

[18] Темам Р. Уравнения Навье-Стокса. Теория и численный анализ. М.: Мир, 1981.

[19] Панагиотопулос П. Неравенства в механике и их приложения. М.: Мир, 1989.

Институт прикладной математики ДВО РАН

Поступило

E-mail: cheb@ipm.marine.su

02.11 .1998 